\title{
Le SIDA murin comme modèle animal du SIDA
}

\section{Paul Jolicœur}

P. Jolicœur : Directeur. Laboratoire de biologie moléculaire, Institut de recherches cliniques de Montréal, 110, avenue des Pins ouest, Montréal, Québec, Canada H2W 1R7 ; département de microbiologie et d'immunologie, faculté de médecine, Université de Montréal, Montréal, Québec, Canada H3C 3 J7 et Department of Experimental Medicine, McGill University,

Le syndrome MAIDS (murine AIDS) est initialement induit par un rétrovirus défectif. Les souris développent une lymphadénopathie et une splénomégalie importantes. Ce syndrome débute par la prolifération de cellules $B$ infectées. Les fonctions des cellules $B$ et aussi celles des cellules $\mathbf{T}$ sont altérées par l'infection, mais la présence de cellules $\mathrm{T} \mathrm{CD4}^{+}$et $\mathrm{B}$ intactes semble nécessaire pour que la maladie se développe. L'agent étiologique est un rétrovirus défectif dont le génome ne contient qu'une seule phase ouverte de lecture codant pour une protéine précurseur Pr60 gas. Cette protéine n'est pas clivée mais elle est myristyllée et phosphorylée. La Pr60 sag semble donc à elle seule responsable du syndrome d'immunodéficience observé. Le syndrome de MAIDS progresse en l'absence de réplication virale c'està-dire sans la réinfection de nouvelles cellules cibles. Ce virus semble se comporter comme un virus oncogénique en induisant directement ou indirectement un lymphome de type $B$. Les cellules $B$ infectées sont capables d'anergiser les cellules $T$ soit en produisant un facteur (cytokine) soit en interagissant elles-mêmes avec d'autres cellules du système immunitaire telles que les cellules $\mathrm{CD}^{+}$. Il semble admis qu'un effet superantigène classique ne peut expliquer la pathogenèse induite par le MAIDS. Ce modèle soulève l'hypothèse d'un rôle important des virus défectifs et de l'infection des cellules $B$ dans le SIDA humain. Il suggère aussi que sa progression ne soit pas nécessairement reliée à la réplication virale.

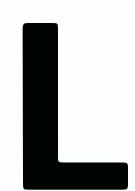

e syndrome d'immunodéficience acquise (SIDA) chez l'humain est une maladie fort complexe qui semble induite par un rétrovirus, le virus d'immunodéficience humain (VIH). En dépit du nombre très con- sidérable de travaux qui ont permis, au cours des huit dernières années, d'accumuler une foule de données sur ce virus, sur son cycle viral et sur ses interactions avec la cellule-hôte [5], nous ne savons toujours pas comment il induit l'immunodéficience sévère 
qui frappe une grande proportion des patients infectés, après une très longue latence. Faute de données expérimentales solides, aucune des nombreuses hypothèses portant sur le mécanisme d'action du VIH [24] ne rallie unaninement la communauté scientifique.

Il semble donc évident que, pour comprendre la pathogénèse du SIDA humain, il faudra procéder comme pour les autres maladies humaines, c'est-à-dire étudier des modèles animaux d'immunodéficience.

\section{Les modèles animaux du SIDA}

Aucun animal n'a encore contracté le SIDA à la suite de l'inoculation du VIH. Seuls le chimpanzé et le lapin sont infectables par le VIH ; mais la réplication virale chez les animaux de ces deux espèces n'entraîne pas d'immunodéficience. Cependant, le virus VIH peut se répliquer chez des souris SCID immunodéficientes et reconstituées avec des cellules hématopoiétiques humaines. $\mathrm{Ce}$ modèle animal pourrait être fort utile pour étudier certains aspects de la réplication virale. Par contre, l'on connaît de nombreux modèles animaux d'immunodéficience induits par des rétrovirus animaux, et qui possèdent unes ou plusieurs propriétés semblables à celle du SIDA humain [7]. Le modèle simien (SAIDS) est certes celui qui se rapproche le plus du syndrome humain tant par ses manifestations cliniques que par son étiologie (un rétrovirus de primate de la famille des lentivirus, comme VIH) [7]. Le syndrome d'immunodéficience du chat (FAIDS), induit par un lentivirus félin, ressemble aussi par certains de ses aspects à la maladie humaine. De plus, il existe plusieurs syndromes d'immunodéficience induits par des rétrovirus qui ne sont pas des lentivirus, tels le rétrovirus simien de type $\mathrm{D}$, les virus défectifs félins ou murins de la leucémie. Ces virus, certes dotés d'une structure génomique différente de celle des lentivirus, induisent des immunodéficiences dont les manifestations ressemblent à celles du SIDA humain. D'autre part, des souris transgéniques exprimant quelques-uns ou tous les gènes du VIH dans des tissus spécifiques ont été produites [16]. Ces sou$m / s n^{\circ} 5$ vol. 9, mai 93

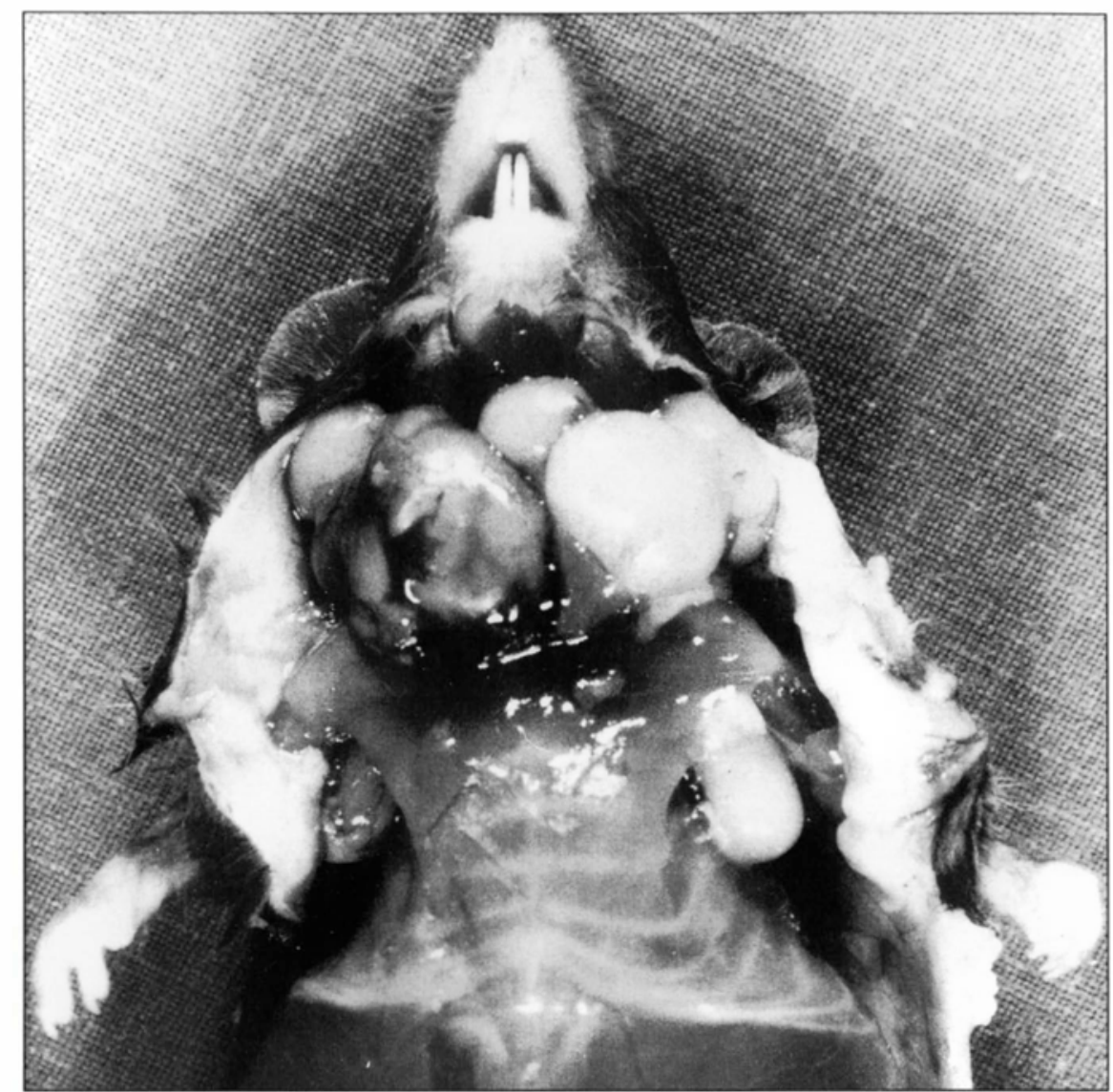

Figure 1. Souris atteinte du MAIDS. Notez la lymphadénopathie.

ris pourraient être fort utiles pour comprendre certains aspects de la relation virus-hôte.

Enfin, de nombreuses mutations, tant chez l'homme que chez la souris, ainsi que de nombreuses maladies bactériologiques, virales, parasitaires ou toxiques entraînent une immunodéficience. Il serait étonnant que le virus VIH utilise une voie métabolique totalement distincte de celle qu'empruntent ces gènes mutés ou ces divers agents pathogènes pour induire une immunodéficience.

Je me propose de revoir ici l'un des modèles du SIDA, le modèle murin (MAIDS) induit par un rétrovirus défectif $[14,15,21]$. Des résultats récents sur la pathogenèse du MAIDS ont permis de formuler de nouvelles hypothèses qui pourraient s'avérer fécondes pour l'étude de l'immunodéficience humaine.

\section{Description du syndrome murin}

La maladie, connue maintenant sous le nom de MAIDS (murine AIDS) [21], a été pour la première fois identifiée dans le laboratoire de Duplan, en France, chez des souris C57BL/6 inoculées avec dés extraits acellulaires de thymomes induits par irradiation aux rayons-X [19]. Au lieu de développer des thymomes, certaines souris présentèrent une lymphadénopathie et une splénomégalie importante (figure 1). En passant à intervalles réguliers, chez la souris, des extraits acellulaires de ces organes hypertrophiés, le groupe de Duplan obtint une souche virale très virulente, capable d'induire la maladie chez près de $100 \%$ des animaux. Ce groupe de recherches, après avoir décrit en détail une anomalie importante des lymphocytes $\mathrm{T}$ et $\mathrm{B}$ et une hypergammaglobulinémie, considéra néanmoins qu'il s'agissait d'un sarcome réticulaire [17]. Ce n'est qu'après le début de l'épidémie du SIDA humain, que des chercheurs américains se rendirent compte que cette maladie de la souris présentait plusieurs des caractéristiques de la 


\section{RÉFÉRENCES}

1. Aziz DC, Hanna $Z$ and Jolicœur $P$. Severe immunodeficiency disease induced by a defective murine leukaemia virus. Nature $1989 ; 338$ : 505-8.

2. Cerny A, Hugin AW, Holmes $\mathrm{KL}$ and Morse MC III. CD4 + $\mathrm{T}$ cells in murine acquired immunodeficiency syndrome : evidence for an intrinsic defect in the proliferative response to soluble antigen. Eur $J$ Immunol 1990 ; 20 : 1577-81.

3. Cerny A, Hügin AW, Hardy RR, Hayakawa K, Zinkernagel RM, Makino M and Morse HC III. B cells are required for induction of $\mathrm{T}$ cell abnormalities in $\mathrm{a}$ murine retrovirus-induced immunodeficiency syndrome. $J$ Exp Med 1990; 171:315-20.

4. Chattopadhyay SK, Morse HC III, Makino M, Ruscetti SK and Hartley HW. Defective virus is associated with induction of murine retrovirus-induced immunodeficiency syndrome. Proc Nall Acad Sci USA 1989 ; 86 : 3862-6.

5. Evans LA and Levy JA. Characteristics of HIV infection and pathogenesis. Biochem Biophys Acta 1989 ; 989 : $237-54$.

6. Fitzpatrick EA, Bryson JS, Rhoads C, Kaplan AM and Cohen DA. T-deficient transmembrane signaling in $\mathrm{CD} 4{ }^{+} \mathrm{T}$ cells of retroviral-induced immune-deficient mice J Immunol 1992 ; 148 : 3377-84.

7. Gardner MB and Luciw PA. Animal models of AIISS. FASEB J. 1989 ; 3 : 2593-606.

8. Ho DD, Moudgil T and Alan M. Quantitation of human immunodeficiency type I in the blood of infected persons. $N$ Engl $J$ Med 1989; 321 : 1621-5.

9. Huang $\mathbf{M}$ and Jolicœur $\mathbf{P}$. Characterization of the gag/fusion protein encoded by the defective Duplan retrovirus inducing murine acquired immunodeficiency syndromes. J Virol. 1990 ; 64 : 5764-72

10. Huang $\mathbf{M}$, Simard $\mathrm{C}$ and Jolicceur $\mathbf{P}$. Immunodeficiency and clonal growth of target cells induced by helper-free defective retrovirus. Science 1989 ; 246 : 1614-7.

11. Huang $\mathbf{M}$, Simard $C$ and Jolicœur. $P$. Susceptibility of inbred strains of mice to murine AIDS (MAIDS) correlates with target cell expansion and high expression of the defective MAIDS virus. J Virol $1992 ; 66$ : maladie humaine $[21,22]$ : une immunodéficience sévère, une hypergammaglobulinémie (augmentation des IgM, IgG3 et IgG2a, mais non pas des IgA ou Ig1), une lymphoprolifération, une susceptibilité aux infections et le développement tardif de lymphomes de type B très agressifs. On est donc en présence d'une immunodéficience sévère. En effet, toutes les fonctions $\mathrm{T}$ mesurées sont anormales : la réponse blastogénique aux mitogènes et aux alloantigènes, la génération de cellules $T$ cytotoxiques, la fonction helper $\mathrm{T}$ pour la production d'anticorps in vitro [17, 22]. Les anomalies des cellules $\mathrm{T}$ semblent être intrinsèques aux cellules $\mathrm{CD} 4^{+}$ (et non aux cellules $\mathrm{CD}^{+}$) et ne résultent pas, semble-t-il d'une altération des fonctions des cellules présentant les antigènes [2]. De plus, il serait possible que les anomalies des cellules $\mathrm{T} \mathrm{CD} 4{ }^{*}$ soient réversibles et ne touchent que certaines voies de signalisation ; en effet, un traitement avec le phorbol ester, PMA (activant la protéine kinase $\mathrm{C}$ ) et l'ionomycin (augmentant $\mathrm{le} \mathrm{Ca}^{2+}$ intracellulaire) stimule leur prolifération [6]. Quant aux cellules B, leurs fonctions sont également anormales comme en témoignent leur réponse faible aux lipopolysaccharides, aux anti- $\mu$, et leur incapacité à produire des anticorps spécifiques en réponse aux antigènes. En outre, des cellules $T$ $\mathrm{CD}^{+}$(mais non pas $\mathrm{T} \mathrm{CD} 8^{+}$) et des cellules $B$ intactes semblent être nécessaires pour que la maladie se développe [3, 28].

Peu après l'inoculation du virus (chez la souris), la forte prolifération cellulaire qui suit affecte principalement la rate et les ganglions, et mène à la destruction de l'architecture normale de ces organes. Plusieurs types cellulaires prolifèrent: cellules $T, B$, macrophages ; néanmoins c'est la prolifération/différenciation des cellules $\mathrm{B}$ qui serait à l'origine de l'hypertrophie de la rate et des ganglions. Par la suite, la majorité des cellules en phase proliférative sont identifiées comme non-T et non-B. En fait, très rapidement, la population des cellules $\mathrm{T} \mathrm{CD}^{+}{ }^{+}$se redistribue et l'on observe l'expansion considérable d'une sous-population marquée par les anticorps monoclonaux SM3G11 et SM6C10 [20]. Or cette population, qui représente une minorité des cellules $\mathrm{T} \mathrm{CD}^{+}{ }^{+}$normales, ne répond pas aux mitogènes, même chez la souris normale. Le sort des autres cellules $\mathrm{T} \mathrm{CD}^{+}{ }^{*}$ demeure inconnu. On remarque certes quelques pertubations des niveaux de certaines lymphokines ou cytokines (notamment l'interféron $\gamma$ ), mais leur rôle, s'il en est un, dans la pathogénèse, n'a pas été établi.

\section{Identification de l'agent étiologique}

L'extrait brut du virus de Duplan, capable de transmettre le MAIDS, contenait plusieurs variants de rétrovirus non défectifs (ayant tous les gènes nécessaires à la réplication, gag, pol et env). Aucun de ces nombreux variants, isolés au cours des ans, n'était capable d'induire la maladie (postulat de Koch). L'on sait maintenant que tous les essais précédents excluaient sélectivement l'agent pathogène. Il a fallu appliquer une méthode d'analyse plus moléculaire, ne faisant appel à aucune sélection biologique pour que notre laboratoire d'abord [1], puis celui du Dr Morse [4], puissent identifier l'agent pathogène et établir qu'il s'agit d'un virus défectif (figure 2). La séquence du génome viral $(4.8 \mathrm{kbp})$ révéla un seul long cadre de lecture dans la région gag, avec la capacité de coder pour une protéine gag précurseur de $60 \mathrm{kd}$ (Pr60"ag) (figure 3) [1]. Le gène gag encode normalement les protéines structurales [p15 (MA), p12, p30 (CA), p10 (NC)] qui sont d'abord synthétisées comme précurseurs (Pr65 ${ }^{\mathrm{gag}}$ ) et par la suite clivées. Cette protéine Pr60 gag est de 95 à $98 \%$ identique à la protéine gag du virus non défectif, sauf dans la région p12. La protéine $\operatorname{Pr} 60^{\text {gag }}$ est facilement détectable dans des fibroblastes transfectés avec le DNA du virus défectif : elle n'est pas clivée, mais elle est myristylée et phosphorylée ; de plus, elle s'attache à la membrane cytoplasmique tout comme la protéine gag normale [9]. Cette protéine, qui semble être la seule protéine encodée par le génome viral, est responsable du syndrome d'immunodéficience observé.

La plupart des virus défectifs connus contiennent un oncogène dérivé du 


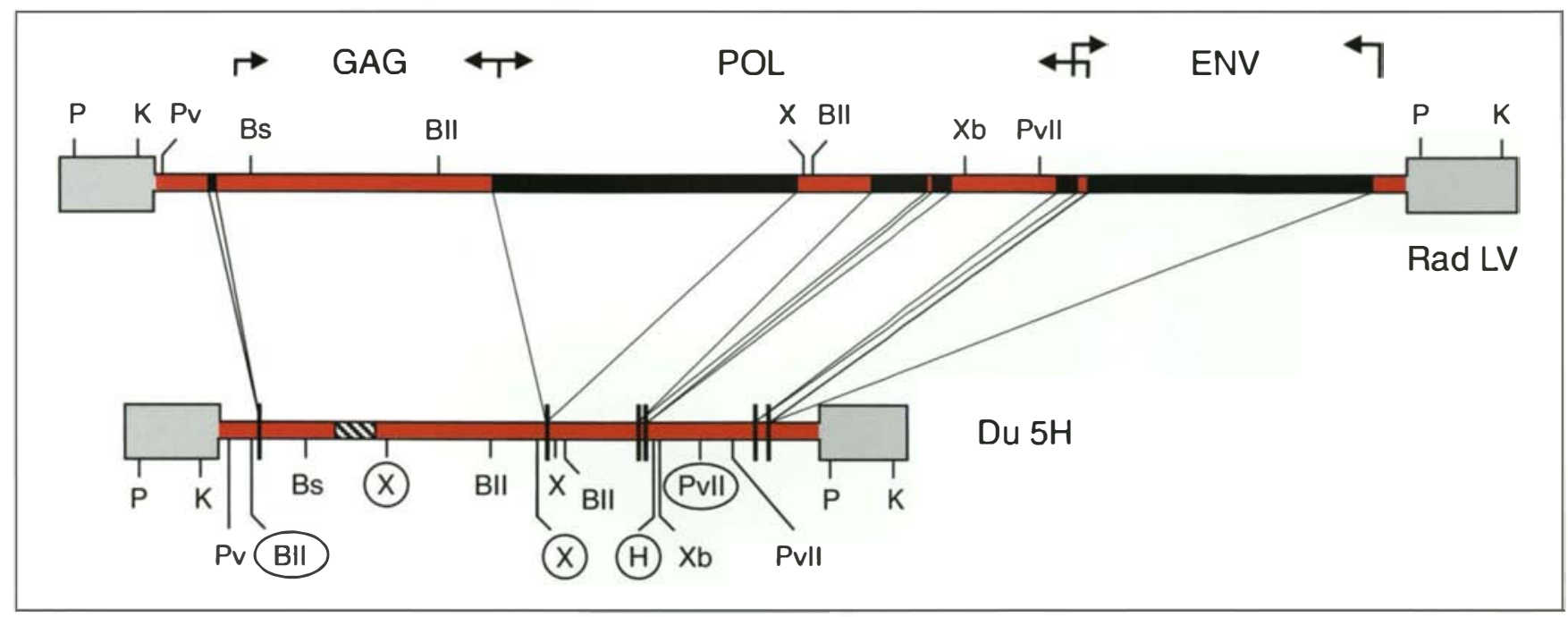

Figure 2. Structure du virus défectif du MAIDS. Le virus défectif du MAIDS (Du5H) est plus court et a perdu une bonne partie des séquences de pol et env (blocs noirs). Le gène gag est intact, mais modifié dans la région p12 (bloc hachuré). Les lettres représentent les endonucléases de restriction utilisées. Le virus auxiliaire (helper) (RadLV) est porteur des trois gènes gag, pol et env.

génome cellulaire. Et il existe peu d'exemples de rétrovirus défectifs n'encodant pas d'oncogènes. Le virus défectif SFFV (spleen focus forming virus) ne contient qu'un gène, env. La structure du virus du MAIDS est donc unique parmi tous les rétrovirus connus.

\section{Rôle de la réplication virale dans l'évolution de la maladie}

Un virus défectif requiert un virus auxiliaire (helper) non défectif pour se répliquer, puisque son génome n'encode pas certains gènes essentiels. Le virus auxiliaire lui fournit en trans les protéines qui lui manquent. Il est possible de construire des stocks de virus défectifs qui ne contiennent pas de virus auxiliaires (helper free). De tels virus sont capables d'infecter une cellule ; par contre, la cellule infectée ne peut produire de virus. En effet, il s'agit d'une infection à un seul cycle viral. Or nous avons observé qu'un stock " helper free " du virus défectif du MAIDS était aussi pathogène qu'un stock contenant un virus non défectif réplicatif [10]. Ce résultat inattendu nous a permis d'étudier le développement de la maladie sans réplication du virus non défectif, c'est-à-dire en absence de bruits de fond, puisque cette réplication n'est pas essentielle à l'appari- tion de la maladie. Dans ces conditions expérimentales, le syndrome du MAIDS, initialement induit par une infection virale, progresse en l'absence de réplication virale, c'està-dire sans la réinfection de nouvelles cellules cibles.

\section{Prolifération clonale des lymphocytes B induite par le virus défectif du MAIDS}

Peu de temps après l'infection d'une souris avec ce virus défectif " helper free ", on observe une prolifération importante des cellules infectées dans la rate et dans presque tous les ganglions [10] (figure 4). Cette prolifération, d'abord polyclonale, devient oligoclonale ou clonale au cours de la phase avancée de la maladie. Parfois, un seul clone envahit tous les ganglions. Fait un peu étonnant, les cellules infectées ne constituent souvent

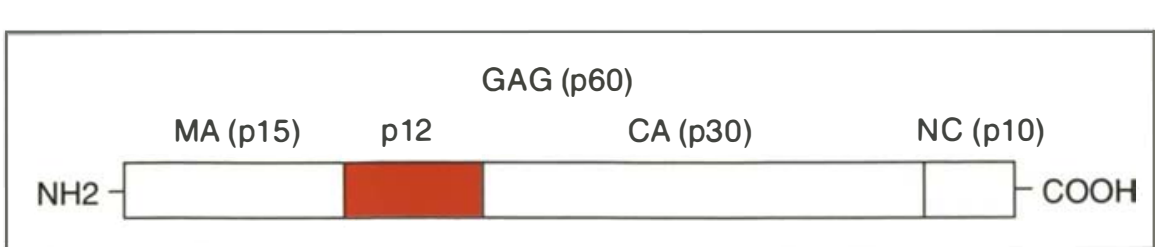

Figure 3. Structure de la protéine gag (Pr609ag) encodée par le virus défectif du MAIDS. Les séquences p15, p30 et p10 sont très homologues aux séquences du virus non défectif. La région $p 12$ (hachurée) du virus défectif est celle qui est la plus divergente des autres séquences p12 des virus non défectifs de la souris.

qu'une minorité (une proportion parfois très faible de l'ordre de $2-5 \%$ ) des cellules des ganglions hypertrophiés. En fait, le nombre de cellules infectées dans un ganglion ne semble pas déterminer la grosseur du ganglion. Un nombre considérable de cellules non infectées semblent être recrutées ou/et semblent proliférer dans ces ganglions. Nous avons récemment pu identifier ces cellules infectées : elles appartiennent à la lignée lymphocytaire $\mathrm{B}$ ( $\mathrm{c} \mathcal{x}$ réarrangé, $\left.\mathrm{B}^{2} 20^{+}\right)[12]$. Nous avons observé, lors d'une étude sur la susceptibilité des différentes souches de souris du MAIDS, que la prolifération de ces cellules infectées est en étroite corrélation avec l'apparition de l'immunodéficience [11].

Le virus défectif du MAIDS se comporte donc comme un virus oncogène et induit, directement ou indirectement, un lymphome de type B. Ce lymphome est, pensons-nous, bénin, 


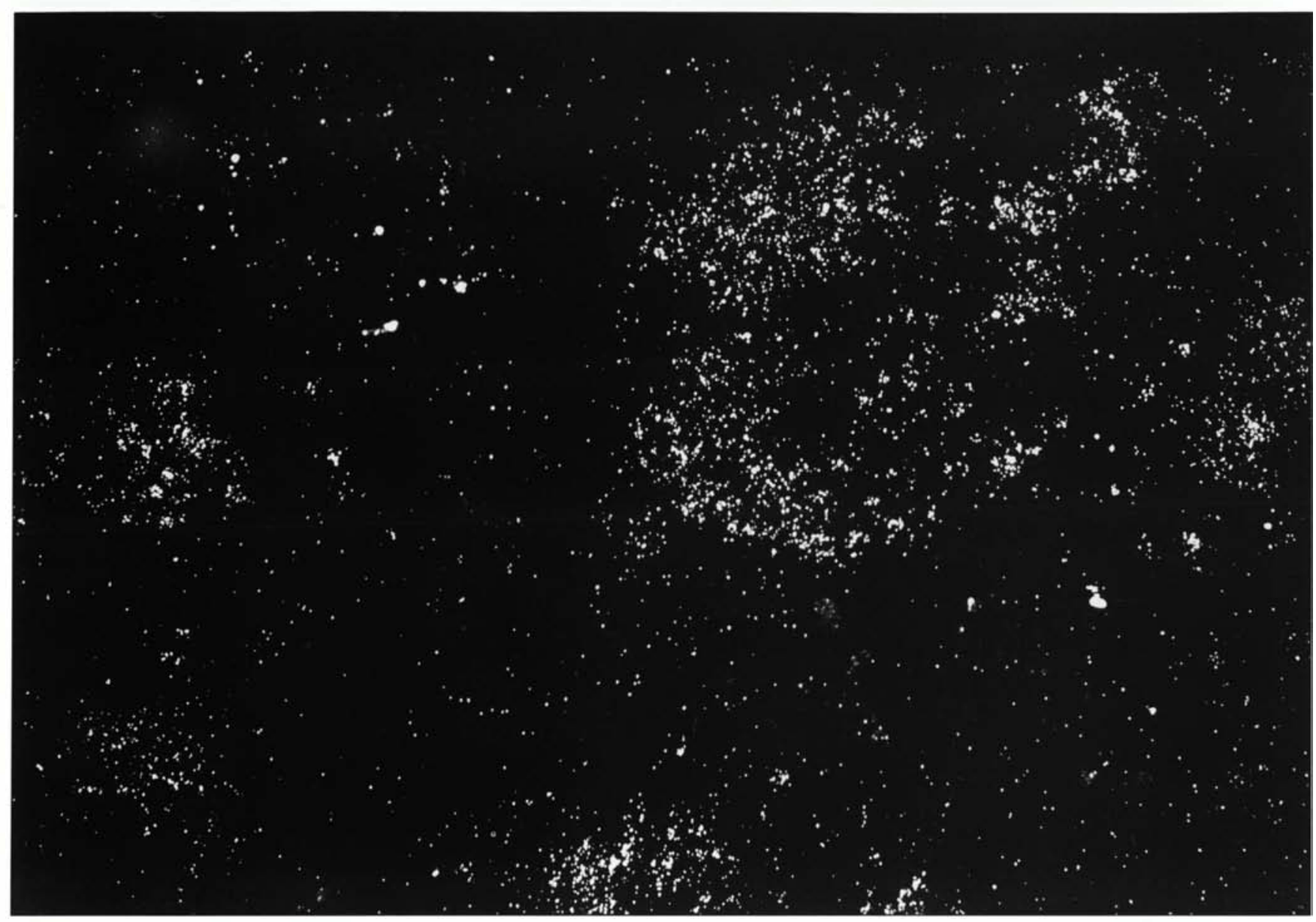

Figure 4. Détection des cellules infectées avec le virus défectif du MAIDS. Une coupe en paraffine d'un ganglion d'une souris infectée 20 jours plus tôt avec le virus défectif du MAIDS. La détection des cellules infectées s'est faite par hybridation in situ avec une ribosonde spécifique à la région U3 du LTR. Champ noir. Noter en blanc, le regroupement des cellules infectées en plusieurs petits clones distincts.

\section{RÉFÉRENCES}

12. Huang M, Simard C, Kay DG and Jolicœur $P$. The majority of cells infected with the defective murine AIDS virus belong to the B-cell lineage. J Virol $1991 ; 65$ : $6562-71$

13. Hugin AW, Vacchio MS and Morse HC III. A virus-encoded " superantigen " in a retrovirus-induced immunodeficiency syndrome in mice. Science 1991; 252: 424-7.

14. Jolicœur P. Murine acquired immunodeficiency syndrome (MAIDS) : an animal model to study the AIDS pathogenesis. FASEB J 1991; 5 : 2398-405.

15. Jolicœur P, Hanna Z, Aziz D, Simard $C$ and Huang $M$. The murine acquired immunodeficiency syndrome (MAIDS) induced by the Duplan strain retrovirus, p. 113-132. In Fan HY, Chen ISY, Rosenberg $\mathrm{N}$ and Sugden W (eds.), Viruses that affect the immune system. American Society

puisque d'une part la prolifération ne semble pas létale et que, d'autre part, les cellules $B$ sont très difficilement transplantables. En revanche, à la suite de la prolifération des cellules $B$ infectées, apparaît une immunodéficience sévère accompagnée d'une lymphoprolifération importante de cellules non infectées (figure 5).

\section{Traitement du MAIDS avec des agents anti-néoplasiques}

Puisque le MAIDS semble d'abord progresser suite à la prolifération des cellules infectées, il nous a paru logique de tenter de traiter cette maladie avec des agents anti-néoplasiques. De tels médicaments, notamment le cyclophosphamide, se sont avérés très efficaces pour prévenir l'apparition du syndrome et pour en induire la régression [26]. Puisque le cyclophos- phamide est un agent immunosuppresseur capable de guérir une immunodéficience, son effet peut paraître ici paradoxal, sauf si l'immunodéficience est d'abord provoquée par la prolifération d'une population cellulaire, phénomène que nous avons en effet observé. En outre, ce résultat confirme à nouveau le rôle important de la prolifération cellulaire dans l'apparition de l'immunodéficience.

\section{Le MAIDS est-il la manifestation d'un effet superantigène ?}

L'une des conséquences importantes de l'infection avec le virus défectif du MAIDS se traduit par l'anergie de presque toutes les cellules $\mathrm{T} \mathrm{CD}^{+}$. Cette anergie pourrait constituer la réponse à un effet superantigène de la protéine Pr60gag. Bien que le virus défectif se comporte comme un onco- 
gène (mitogène) dans les cellules lymphocytaires $B$, il se peut que la prolifération que l'on constate soit déclenchée par des lymphocytes T $\mathrm{CD}^{+}{ }^{+}$qui réagiraient ainsi à la présence de la protéine Pr60gag présentée par les lymphocytes B. Un effet superantigène observé avec unc lignéc de lymphocytes $B$ dérivée des souris atteintes du MAIDS [13] accrédite cette hypothèse, quoique, pour l'instant, cet effet n'ait pas été décelé par un autre groupe de recherche [23]. La protéine Pr60gag est myristylée; il est peu probable qu'elle soit exposéc à la face externe de la membrane cellulaire. Il n'est pas non plus exclu que l'effet observé provienne des Mls possiblement induits dans les cellules étudiées. De plus, les superantigènes ne mènent en général qu'à la délétion ou à l'anergie de souspopulations de cellules $\mathrm{T} V \beta$ et non pas à celle de toutes les cellules $T$ $V \beta$. Enfin, des souris porteuses de délétions des gènes $\mathrm{V} \beta$ normalement exprimés à la surface des cellules $\mathrm{V} \beta$ impliquées dans le MAIDS, sont susceptibles au MAIDS. Cette observation ne peut aller de paire avec l'idée que la délétion de sous-populations cellulaires $\mathrm{V} \beta$ joue un rôle majeur dans la pathogenèse du MAIDS. Il est donc maintenant admis qu'un effet superantigène classique (type Mls) ne peut expliquer la pathogenèse du MAIDS. L'effet de la protéine Pr60gag chez l'animal est, pensons-nous, assez différent d'un effet superantigène classique. Si la

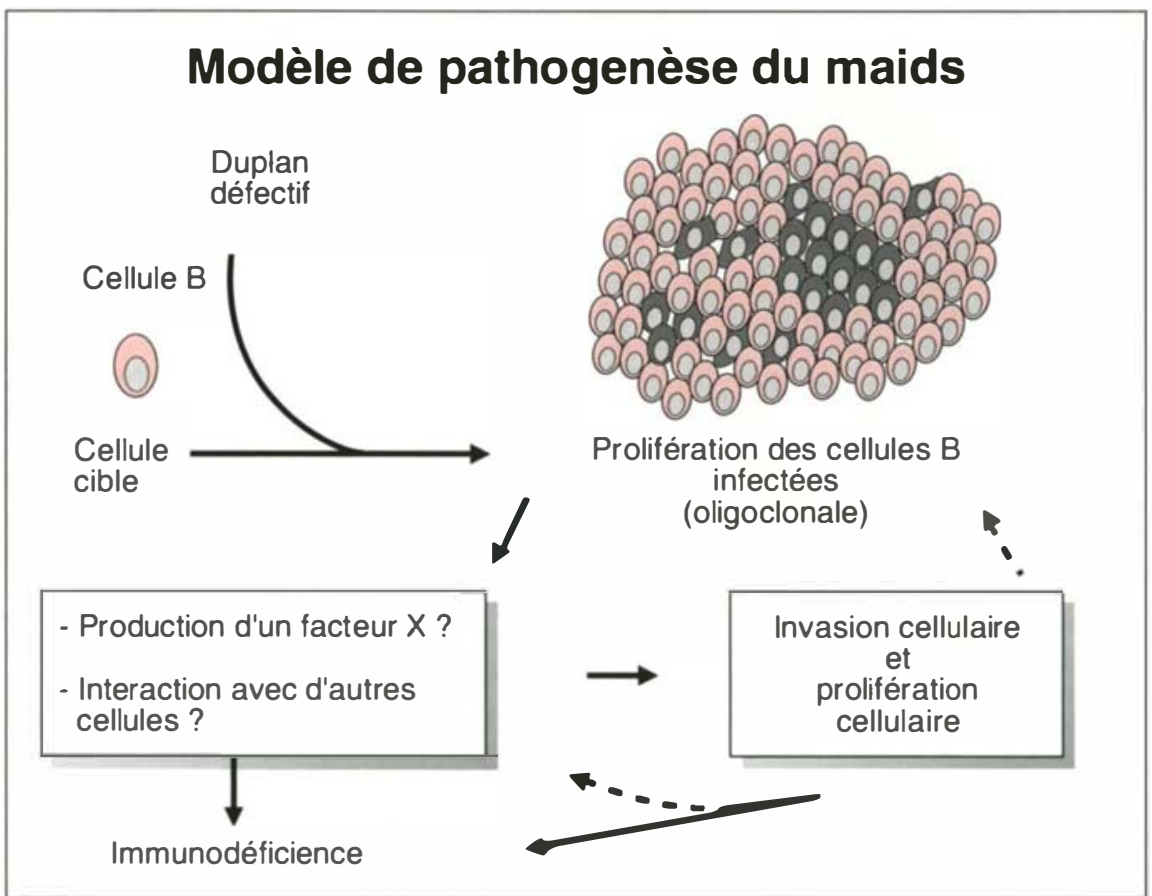

Figure 5. Modèle de pathogenèse du MAIDS. A la suite de l'infection par le virus défectif du MAIDS, les cellules lymphocytaires B prolifèrent en petits clones. Ceux-ci ne représentent souvent qu'une minorité des cellules du ganglion hypertrophié. D'autres cellules non infectées llymphocytes $T$, B et macrophages) prolifèrent aussi dans ces ganglions ou/et y sont recrutées. Curieusement, le degré de lymphadénopathie n'est pas déterminé par le nombre de cellules infectées dans un ganglion donné. La prolifération de ces cellules infectées mène éventuellement à l'apparition d'une immunodéficience, principalement visible par l'anergie des cellules $T$. Nous postulons que les cellules $B$ infectées sont capable $d^{\prime}$ anergiser les cellules $T$, soit en produisant un facteur " $x$ " (peut-être une cytokine), soit en interagissant elles-mêmes avec d'autres cellules du système immunitaire (peut-être avec les cellules T CD4+). La sécrétion de ce facteur par les cellules infectées et/ou leur interaction avec d'autres cellules, peut elle-même entraîner dans le ganglion, le recrutement et la prolifération de nouvelles cellules qui pourraient aussi participer à l'émergence de l'immunodéficience.

$\mathrm{m} / \mathrm{s} n^{\circ} 5$ vol. 9 , mai 93

protéine Pr60gag agit par un mécanisme analogue à celui des superantigènes, elle mérite d'être classée dans une catégorie distincte d'antigènes, que nous avons proposé de nommer des "ultra-antigènes " [12]. Des expériences en cours devraient déterminer si le MAIDS représente la réponse du système immunitaire à un antigène peut-être très spécial selon un modèle impliquant la reconnaissance de l'antigène dans une structure comprenant sans doute des molécules de classe II ou s'il constitue plutôt une dérégulation de la croissance cellulaire d'une sous-population lymphocytaire $B$, entraînant une perturbation du système immunitaire [peut-être par secrétion de cytokine(s)] ; un tel modèle n'implique pas nécessairement une reconnaissance de l'antigène par les cellules $T$.

\section{MAIDS est-il un modèle animal de maladies humaines}

Il est toujours difficile de déterminer jusqu'à quel point un modèle animal d'une maladie reflète une maladie humaine. La plupart des bons modèles animaux sont semblables, mais non identiques, à une maladie humaine. Le MAIDS n'échappe pas à ce principe. Plusieurs des manifestations du MAIDS sont assez semblables à celles que l'on rencontre dans le SIDA humain pour permettre de croire que l'étude de ce modèle murin puisse être utile à la compréhension de certaines manifestations du SIDA. De plus, des résultats récents laissent croire que ce modèle pourrait éclairer certaines autres maladies humaines.

A) MAIDS et SIDA humain. En incitant les chercheurs à formuler de nouvelles questions et à poser de nouvelles hypothèses sur le SIDA humain, le modèle de MAIDS a incontestablement contribué à revoir le rôle des virus défectifs et de la réplication virale ainsi qu'à susciter une étude plus poussée de la présence et de la nature de cellules cibles spécifiques dans le SIDA humain.

Le simple fait que deux rétrovirus défectifs animaux (du groupe des non-lentivirus) induisent le SIDA murin (MAIDS) ou félin (FAIDS) laisse supposer qu'un virus défectif 


\section{RÉFÉRENCES}

16. Jolicœur $\mathrm{P}$, Laperrière $\mathrm{A}$ and Beaulieu N. Efficient production of human immunodeficiency virus proteins in transgenic mice. J Virol 1992 ; 66 : 3904-8.

17. Legrand E, Dalculsi R and Duplan JF Characteristics of the cell populations involved in extra-thymic lymphosarcoma induced in C57BL/6 mice by RadLV-Rs. Leuk Res $1981 ; 5: 223-33$.

18. Meyerhans A, Cheynier R, Albert J, Seth M, Kwok S, Sninsky S, MorfeldtManson L, Asjo B and Wain-Hobson S. Temporal fluctuations in HIV quasispecies in vivo are not reflected by sequential HIV isolations. Cell 1989 ; 58 : 901-10.

19. Mistry PB and Duplan JF. Propriétés biologiques d'un virus isolé d'une radioleucémie C57BL. Bull Cance (Paris) 1973 ; 60 : 287-300.

20. Morse III HC, Yetter RA, Via CS, Hardy RR, Cerny A, Hayakawa K, Hugin AW, Miller MW, Holmes KL and Shearer GM. Functional and phenotypic alterations in $\mathrm{T}$ cell subsets during the course of MAIDS, a murine retrovirus-induced immunodeficiency syndrome. J Immunol $1989 ; 143: 844-50$.

21. Mosier DE. Animal models for retrovirus-induced immunodeficiency disease. Immunol Invest $1986 ; 15: 233-61$.

22. Mosier DE, Yetter RA and Morse HC III. Retroviral induction of acute lymphoproliferative disease and profound immunosuppression in adult C57BL/6 mice. J Exp Med 1985 ; 161 : 766-84.

23. Muralidhar G, Koch S, Haas $M$ and Swain SL. CD4 $T$ Cells in murine acquired immunodeficiency syndrome : polyclonal progression to anergy. J Exp Med 1992 ; $175: 1589-99$.

24. Rosenberg ZF and Fauci AS. The immunopathogenesis of HIV infection. Adv Immunol $1989 ; 47$ : 377-431.

25. Schnittman SM, Psallidopoulos MC, Lane HC, Thompson L, Baseler M, Massari F, Fox CH, Salzman NP and Fauci AS. The reservoir for $\mathrm{HIV}_{-1}$ in human peripheral blood is a $\mathrm{T}$ cell that maintains expression of CD4. Science 1989; 245 : 305-8.

26. Simard C and Jolicour P. The effect of anti-neoplastic drugs on murine acquired immunodeficiency syndrome. Science 1991 ; $251: 305-8$.

27. Weiss RA. Defective viruses to blame ? Nature 1989 ; 338-458.

28. Yetter RA, Buller RML, Lee JS, Elkins KL, Mosier DE, Fredrickson TN and Morse HC III. CD4 ${ }^{+} \mathrm{T}$ cells are required for development of a murine retrovirusinduced immunodeficiency syndrome
VIH puisse intervenir dans la pathogenèse [27]. Or la présence de plusieurs classes de virus défectifs VIH est maintenant bien documentée chez les patients atteints du SIDA [18]. Et il semble que ce soit également le cas chez les primates infectés avec SIV. Cependant, il faut admettre que le rôle (s'il y en a un) de ces virus défectifs dans l'induction et/ou la progression de la maladie humaine demeure encore inconnu [27].

Comme nous l'avons énoncé plus haut, le MAIDS peut progresser en l'absence de réplication virale, c'està-dire sans la réinfection de nouvelles cellules. Cette observation soulève l'hypothèse que la réinfection de nouvelles cellules par VIH, ne soit pas non plus nécessaire à la progression du SIDA. Il n'existe, en effet, aucune expérience convaincante qui établisse un lien causal entre la progression du SIDA et la réplication du VIH. Les données disponibles montrent tout simplement que les titres de virus infectieux et le nombre de cellules infectées augmentent de façon significative à mesure que l'immunodéficience progresse $[8,25]$. Or la poussée de la réplication virale n'est peutêtre que la conséquence d'un affaissement du système immunitaire plutôt que sa cause. Il est, en effet, bien connu que de nombreux autres micro-organismes (incluant d'autres virus) se répliquent beaucoup mieux au cours de la phase avancée du SIDA.

Une observation supplémentaire laisse supposer que la réplication du VIH joue un rôle restreint dans la progression du SIDA. Il s'agit de l'anergie précoce des cellules $\mathrm{T}$, détectée au début de la maladie bien avant la déplétion des cellules T $\mathrm{CD}^{+}[5$, 24] au moment où les titres plasmatiques de VIH et le nombre de cellules sanguines infectées sont toujours très bas $[8,25]$.

Cette discussion est loin d'être académique. En effet, presque toutes les stratégies thérapeutiques contre le SIDA sont fondées sur l'hypothèse que la réplication du VIH entraîne une progression du SIDA; elles visent donc d'abord à bloquer cette réplication. Si la réplication accélérée du VIH est la conséquence plutôt que la cause de la progression du SIDA, il est clair que ces médica- ments n'auront aucun effet bénéfique sur cette progression. Et un tel résultat fournirait une preuve assez convaincante de l'absence du rôle de la réplication de VIH dans l'évolution de la maladie. En fait, un tel essai clinique m'apparaît comme la seule façon de vérifier cette hypothèse, pourvu que le médicament anti-VIH utilisé soit hautement spécifique et n'ait aucun effet sur le métabolisme ou la prolifération cellulaire. Cette condition est essentielle. L'AZT et le ddI, deux inhibiteurs très efficaces de la réplication du VIH largement employés en clinique contre le SIDA, ne sont pas complètement spécifiques. Il ne serait donc pas étonnant (et ce n'est là qu'une hypothèse) que leur effet modérément bénéfique sur la progression de l'immunodéficience dépende non seulement de leur effet anti-viral, mais aussi, et peut-être davantage de leur effet cellulaire.

Dans le modèle murin MAIDS, il semble qu'une population cellulaire (appartenant à la lignée lymphocytaire B) soit spécifiquement infectée et que cette infection entraîne une modification importante de la cellule, c'est-à-dire une prolifération. La plupart des rétrovirus ciblent ainsi une population bien définie chez l'animal. Il se peut donc que le virus VIH cible aussi une population dont l'infection soit nécessaire (et possiblement suffisante) pour induire le SIDA. Il se peut même que cette population ne soit pas l'une des populations infectées déjà identifiées (cellules $\mathrm{T} \mathrm{CD}^{+}{ }^{+}$, monocytes sanguins, cellules de Langerhans, cellules dendritiques, macrophages et cellules microgliales). Il se peut aussi que cette population soit très rare et localisée dans des organes ou des tissus très spécifiques et n'ait pas encore été reconnue. La prolifération ou la transactivation de certains des gènes de cette population pourrait peut-être être responsable de tout le syndrome observé. Comme dans le MAIDS, cette population pourrait même appartenir à la lignée lymphocytaire B. Plusieurs auteurs ont signalé le rôle important de l'interaction des lymphocytes B avec le VIH [5, 24]. B) MAIDS et autres maladies humaines: Puisque le MAIDS semble d'abord débuter par la prolifération très particulière de cellules infectées apparte- 
nant à la lignée lymphocytaire B, cette maladie de la souris pourrait aussi servir de modèle à d'autres maladies humaines. Par exemple, les cellules de Reed-Sternberg, caractéristiques des lymphomes de Hogkins et qu'on pense être des cellules malignes, ne constituent qu'une très faible proportion de la masse ganglionaire, tout comme les cellules infectées dans le syndrome de MAIDS. Dans ces deux maladies, de nombreuses cellules réactives, apparemment normales, prolifèrent au sein même des ganglions porteurs de cellules " transformées " et d'autres y sont probablement recrutées.

Le MAIDS ressemble aussi à d'autres immunodéficiences associées à des syndromes lymphoprolifératifs, comme le syndrome de lymphadénopathie angio-immunoblastique avec dysprotéinurie, le syndrome lymphoprolifératif récessif lié au chromosome $\mathrm{X}$ et enfin, sous certains aspects, la mononucléose qui se développe à la suite de l'infection des lymphocytes B par le virus d'Epstein-Barr. Il ne serait donc pas surprenant qu'une étude plus poussée du MAIDS serve à une meilleure compréhension de la pathogenèse de ces maladies humaines caractérisées par une immunodéficience importante.

\section{Conclusion}

L'anergie des lymphocytes $\mathrm{T}$, observée dans le MAIDS par suite de l'infection de cellules appartenant à la lignée lymphocytaire $\mathrm{B}$, représente un syndrome très intéressant d'immunodéficience. Sa compréhension devrait non seulement aider les recherches sur le SIDA humain, mais encore éclairer d'autres immunodéficiences humaines associées à des syndromes lymphoprolifératifs.

\section{Remerciements}

Nos travaux bénéficient du soutien financier du Conseil de Recherches Médicales du Canada et de l'Institut National du Cancer du Canada. Je remercie mon collègue Rafik Sékaly et tous les membres de mon laboratoire, en particulier Carole Simard, Ming Huang, Denis G. Kay, Doug Aziz et Zaher Hanna pour les nombreuses discussions intéressantes que nous avons eues à propos du MAIDS. Je remercie Marie Bernier pour la préparation de ce manuscrit.

\section{Summary}

Murine AIDS as an animal model for human AIDS

The MAIDS (murin AIDS) syndrome is induced by a defective retrovirus. The syndrome starts by the proliferation of infected B cells and affected mice develop lymphadenopathy and splenomegaly. The functions of $\mathrm{B}$ cells as well as those of $\mathrm{T}$ cells are altered by the infection but $\mathrm{CD} 4{ }^{+} \mathrm{T}$ cells and normal $B$ cells appear required for development of the disease. The etiologic agent is a defective retrovirus which contains a single open reading frame encoding the Pr60gag protein precursor. This protein is not processed by cleavage but it is myristylated and phosphorylated. Thus, Pr60gag appears entirely responsible for the immunodeficiency syndrome. The MAIDS syndrome progresses even in the absence of viral replication, that is, without infection of new target cells. This virus behaves as an oncogenic virus which induces directly or indirectly a B cell lymphoma. Infected $B$ cells can anergize $T$ cells either by producing a growth factor (cytokine) of by interaction with other cells of the immune system, like $\mathrm{CD}^{+}{ }^{+}$cells. A classical superantigen effect does not appear to be responsible for the MAIDS pathogenesis. This model suggests that defective viruses and $B$ cell infection might be involved in human AIDS and that viral replication might not be essential for progression of the disease.
TIRÉS A PART

P. Jolicour 